\title{
TLR2 inhibition ameliorates the amplification effect of LPS on lipid accumulation and lipotoxicity in hepatic cells
}

\author{
Liting Zhang ${ }^{1,2,3 \#}$, Zehui Xie ${ }^{4 \#}$, Hongmiao $\mathrm{Yu}^{5}$, Haoxuan $\mathrm{Du}^{6}$, Xuqiao Wang ${ }^{7}$, Jiazheng $\mathrm{Cai}^{7}$, Yingfei Qiu ${ }^{7}$, \\ Rui Chen ${ }^{7}$, Xiaofeng Jiang ${ }^{7}$, Zelin Liu ${ }^{7}$, Yi Li ${ }^{1,7}$, Tuo Chen ${ }^{1,2}$ \\ ${ }^{1}$ State Key Laboratory of Cryospheric Science, Northwest Institute of Eco-Environment and Resources, Chinese Academy of Sciences, Lanzhou, \\ China; ${ }^{2}$ University of Chinese Academy of Sciences, Beijing, China; ${ }^{3}$ Department of Infectious Diseases, The First Hospital of Lanzhou University, \\ Lanzhou, China; ${ }^{4}$ The First School of Clinical Medicine, Lanzhou University, Lanzhou, China; ${ }^{5}$ Children's Hospital and Institute of Biomedical \\ Sciences, Fudan University, Shanghai, China; ${ }^{6}$ The First Clinical Medical College of Lanzhou University, Lanzhou, China; ${ }^{7}$ School/Hospital of \\ Stomatology, Lanzhou University, Lanzhou, China \\ Contributions: (I) Conception and design: Y Li, L Zhang; (II) Administrative support: None; (III) Provision of study materials or patients: None; (IV) \\ Collection and assembly of data: Z Xie, H Yu; (V) Data analysis and interpretation: X Wang, H Du; (VI) Manuscript writing: All authors; (VII) Final \\ approval of manuscript: All authors. \\ "These authors contributed equally to this work. \\ Correspondence to: Yi Li, PhD. State Key Laboratory of Cryospheric Science, Northwest Institute of Eco-Environment and Resources, Chinese \\ Academy of Sciences, Lanzhou 730000, China; School of Stomatology, Lanzhou University, Lanzhou 730000, China. Email: liyilz@163.com; Tuo \\ Chen, PhD. State Key Laboratory of Cryospheric Science, Northwest Institute of Eco-Environment and Resources, Chinese Academy of Sciences, \\ Lanzhou 730000, China; University of Chinese Academy of Sciences, Beijing 100049, China. Email: chentuo@lzb.ac.cn.
}

Background: Gut microbiome dysbiosis is related to the pathogenesis of nonalcoholic fatty liver disease (NAFLD), and the role of toll-like receptor 2 (TLR2) in its molecular mechanism is controversial. Here, we investigated the effects and mechanisms of Escherichia coli-derived lipopolysaccharide (LPS) on lipid accumulation and lipotoxicity in palmitic acid (PA)-treated L02 cell as an NAFLD cell model, and the role of TLR2 in this process.

Methods: Oil red O staining assay and free fatty acid (FFA) content test were performed to determine the effects of LPS on lipid accumulation in a PA-induced NAFLD cell model with or without TLR2 inhibition. The levels of IL- 6 and TNF- $\alpha$ were measured to investigate inflammation conditions. Hoechst 33342 staining assay and Caspase-3 activity assay were used to test cell apoptosis, and the expression levels of proteins in the IRS1/PI3K/AKT signaling pathway, TLR2/MyD88/IKK $\alpha / \mathrm{NF}-\kappa \mathrm{B}$ signaling pathway, and mitochondrion-dependent apoptotic signaling pathway were detected using Western blot.

Results: Lipid accumulation, pro-inflammatory cytokine release, and cell apoptosis with high levels were observed in the PA-induced NAFLD cell model, and LPS aggravated these processes. Whereas TLR2 inhibition could significantly ameliorate PA-induced and LPS-amplified lipid accumulation, inflammatory, and cell apoptosis, it had no significant effect on L02 cells treated with LPS alone.

Conclusions: These results were confirmed by activation or inhibition of the IRS1/PI3K/AKT signaling pathway, TLR2/MyD88/IKK $\alpha / \mathrm{NF}-\kappa \mathrm{B}$ signaling pathway, and mitochondrion-dependent apoptotic signaling pathway, and were reflected by changes on their proteins expression. TLR2 is involved in PA-induced lipid accumulation and lipotoxicity in L02 cells, which could be aggravated by LPS, although LPS-induced amplification might not be through direct interaction with TLR2.

Keywords: Toll-like receptor 2; lipopolysaccharide (LPS); nonalcoholic fatty liver disease (NAFLD); lipid accumulation; inflammation

Submitted Jul 08, 2021. Accepted for publication Sep 10, 2021.

doi: 10.21037/atm-21-4012

View this article at: https://dx.doi.org/10.21037/atm-21-4012 


\section{Introduction}

Nonalcoholic fatty liver disease (NAFLD) is one of the main causes of liver transplantation, affecting 20-30\% of the global population (1-3). NAFLD refers to excess triglyceride accumulation in the liver of patients with no history of alcohol abuse or other causes of secondary hepatic steatosis. Lipid accumulation increases the concentration of toxic lipids and lipid derivatives, promoting inflammation, mitochondrial dysfunction, oxidative stress, and cell death, known as lipotoxicity (4). Lipid accumulation and lipotoxicity play core roles in the "Two-Hit Hypothesis", which is accepted as explaining the pathogenesis of NAFLD (5). However, the "Multiple-Hit Hypothesis" implicates that the development and progression of NAFLD is influenced by multiple parallel factors, such as obesity, insulin resistance (IR), dysfunction of adipose tissue, nutritional factors, gut microbiota, and genetic factors $(2,6)$. Compared with the "Two-Hit Hypothesis", the "Multiple-Hit Hypothesis" imbues a more comprehensive explanation of the molecular mechanism of NAFLD. However, the mechanism of each parallel factor on complex human NAFLD remains unclear.

Gut microbiomes are one of the factors in the "MultipleHit Hypothesis", and are considered to be a critical factor in the development and progression of NAFLD by preclinical and clinical studies $(2,6)$. Gut microbiome dysbiosis or altered intestinal permeability may promote the translocation of microbial or microbial metabolites to the portal vein circulation, which is called the gut-liver axis $(4,5)$. In addition, previous studies have shown that oral microbiome dysbiosis can also affect the progression of hepatic disease through the oral-gut-liver axis $(7,8)$. In response to bacteria-derived molecules, Toll-like receptors (TLRs), which are components of the innate immune system, are activated in the liver $(9,10)$. TLR4 and TLR9 are activated by the lipopolysaccharide (LPS), which is the active component of endotoxin from Gram-negative bacteria, and bacterial DNA, respectively, to induce inflammasomes and pro-inflammatory factors, thereby promoting the progress of NAFLD. TLR5 is activated by bacterial flagellin, which promotes bacterial clearance and protects hepatocytes from high-fat diet-induced liver disease. TLR2 can be activated by cell wall components of Gram-positive bacteria, and the downstream signal is activated by the adaptor molecule myeloid differentiation

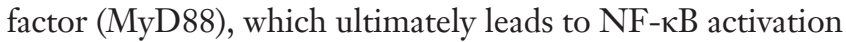
and induction of pro-inflammatory cytokines (10). However, the role of TLR2 in the pathogenesis of NAFLD is controversial. Szabo et al. suggested a methionine-choline deficient diet could induce nonalcoholic steatohepatitis (NASH), and liver injury was more severe after LPS challenge in TLR2-deficient mice, indicating TLR2mediated signals had protective effects in liver injury (11). By contrast, choline-deficient amino acid-defined dietinduced NASH was suppressed in TLR2-deficient mice (3), and periodontal infection of Porphyromonas gingivalis (P.g.) aggravated high fat diet-induced NASH through the P.g.LPS/TLR2 pathway in mice (12). Thus, the role of TLR2 in NAFLD requires further investigation.

In our previous study, palmitic acid (PA)-treated L02 cells served as an NAFLD cell model in vitro. While Escherichia coli-derived LPS activated the TLR4/NF- $\kappa \mathrm{B}$ signaling pathway to promote lipid deposition and cell damage, the role of TLR2 in this process was not explored (13). In the present study, we conducted further research on this point and found that TLR2 was involved in PA-induced lipid accumulation and lipotoxicity, which could be aggravated by LPS, but LPS-induced amplification might not be through direct interaction with TLR2. We present the following article in accordance with the MDAR reporting checklist (available at https://dx.doi.org/10.21037/atm-21-4012).

\section{Methods}

\section{Cell culture}

Human L02 cells were obtained from the Chinese Academy of Sciences Cell Bank (Shanghai, China) and cultured in Roswell Park Memorial Institute 1640 medium (GIBCO, invitrogen, USA) containing $10 \%$ (v/v) fetal bovine serum (FBS) (Invitrogen, Carlsbad, CA, USA), penicillin $(100 \mathrm{U} / \mathrm{mL})$, and streptomycin $(100 \mu \mathrm{g} / \mathrm{mL})$ at $37^{\circ} \mathrm{C}$ in a humidified atmosphere with $5 \% \mathrm{CO}_{2}$. The medium was replaced every $2-3$ days, and the cells were passaged as they grew to $80 \%$ confluence.

\section{Targeted TLR2 inbibition}

Three small interfering RNA oligonucleotides targeting TLR2 (siRNA-TLR2) and negative control nonsensesiRNA oligonucleotide (siRNA-N.C.) were purchased from GenePharma (GenePharma, Shanghai, China). The L02 cells were cultured in Opti-MEM ${ }^{\circledR}$ I medium (GIBCO, Invitrogen, USA) without FBS and antibiotic before 24 h. Lipofectamine ${ }^{\mathrm{TM}} 2000$ (Life Technologies, Gaithersburg, USA) and RNAi molecule (20 pg) were each 
separately diluted in Opti-MEM ${ }^{\circledR}$ I medium without FBS. Samples were then stirred gently, incubated for $5 \mathrm{~min}$ at room temperature, then combined. After another $20 \mathrm{~min}$ at room temperature for incubation, the RNAi moleculeLipofectamine ${ }^{\mathrm{TM}} 2000$ complexes were added to each wellcontaining cell and medium. Cells were then cultured at $37^{\circ} \mathrm{C}$ in a $\mathrm{CO}_{2}$ incubator for $6 \mathrm{~h}$, when the complete medium was replaced. Transfected L02 cells were incubated for $24 \mathrm{~h}$ at $37^{\circ} \mathrm{C}$, then collected to detect protein expression.

\section{Establishment of the PA-induced NAFLD cell model}

L02 cells were treated with $0.4 \mathrm{mM}$ PA (Sigma-Aldrich, MO, USA) for $24 \mathrm{~h}$ after transfection, and $800 \mathrm{ng} / \mathrm{mL}$ Escherichia coli-derived LPS (Sigma-Aldrich, MO, USA) was used to challenge the NAFLD cell model. A control group, siRNA-N.C. group, siRNA-N.C. + LPS group, siRNATLR2 + LPS group, siRNA-N.C. + PA group, siRNATLR2 + PA group, siRNA-N.C. + LPS + PA group, and siRNA-TLR2 + LPS + PA group were established to test the model.

\section{Oil red $O$ staining assay}

Intracellular lipid accumulation was examined using an oil red $\mathrm{O}$ staining assay kit (Jiancheng Bioengineering Institute, Nanjing, China). Cells were fixed by $3.7 \%$ paraformaldehyde at room temperature then removed after 15 min. After washing with PBS three times, oil red O (oil red reserve: diluent $=5: 2$, filtered with filter paper) was applied and the cells were incubated for $15 \mathrm{~min}$. Working fluid was then discarded, the coverslips rinsed three times with warm water $\left(37^{\circ} \mathrm{C}\right)$, and the sample was stained with hematoxylin for 5 minutes and rinsed with water. Cells were imaged using a microscope (ix-81, Olympus, Tokyo, Japan).

\section{Free fatty acid (FFA) assay}

An FFA assay was used to determine the metabolism of the fatty acid. Cells were washed twice with cold PBS and lysed in ice-cold RIPA buffer (Solarbio, Beijing, China) containing $1 \mathrm{mM}$ phenylmethylsulfonyl fluoride (Beyotime, Shanghai, China) for $10 \mathrm{~min}$. After centrifuging the lysates at $12,000 \mathrm{rpm}$ and at $4{ }^{\circ} \mathrm{C}$ for $15 \mathrm{~min}$, the supernatants were collected and used to detect FFA according to the manufacturer's instructions (Jiancheng Bioengineering Institute, Nanjing, China).

\section{Enzyme-linked immunosorbent assay (ELISA)}

The FBS-free culture medium was collected and measured the concentration of protein using a BCA protein assay kit (Cwbio, Beijing, China). An ELISA assay was then performed to assess the levels of IL- 6 and TNF- $\alpha$ using commercial kits (eBioscience, San Diego, CA, USA). The optical densities were measured at $450 \mathrm{~nm}$ in a microplate reader (model ELx800, Biotek, Winooski, Vermont).

\section{Hoechst 33342 staining assay}

Cells were washed with PBS twice and fixed by $3.7 \%$ paraformaldehyde at room temperature for $15 \mathrm{~min}$. Subsequently, the coverslips were washed three times with PBS and $1 \mathrm{mM}$ Hoechst 33342 (Solarbio, Beijing, China), then incubated for $15 \mathrm{~min}$ at $37^{\circ} \mathrm{C}$ in the dark. Apoptotic cells with clear condensation and small bright nuclei were visualized under a microscope (ix-81, Olympus, Tokyo, Japan).

\section{Caspase-3 activity assay}

Total protein was collected and used to detect Caspase-3 activity, which is a marker of cell apoptosis, and its activity was measured using a commercial kit (Nanjing Jiancheng Bioengineering Institute, Nanjing, China). Briefly, the supernatants of protein lysate were mixed with Ac-DEVDpNA for $4 \mathrm{~h}$ in $37^{\circ} \mathrm{C}$ and optical densities were then measured at $405 \mathrm{~nm}$ in a microplate reader (model ELx800, Biotek, Winooski, Vermont).

\section{Western blot analysis}

Cell lysates were prepared with RIPA buffer (Solarbio, Beijing, China) containing $1 \mathrm{mM}$ phenylmethylsulfonyl fluoride (Beyotime, Shanghai, China), and protein concentrations were quantified using a BCA protein assay kit (Cwbio, Beijing, China). Equal amounts of protein were loaded onto $10 \%$ sodium dodecyl sulfate-polyacrylamide gels for protein separation, and the fractionated protein then transferred to polyvinylidene difluoride membranes (Millipore, Billerica, MA, USA). After blocking, the membranes were incubated with the primary antibodies $\beta$-Actin at 1:1,000, insulin receptor substrate 1 (IRS1) at 1:1,000, phosphoinositide 3-kinase (PI3K) at 1:1,000, AKT serine/threonine kinase (AKT) at 1:1,000, phosphorylated$\mathrm{AKT}(\mathrm{p}-\mathrm{AKT})$ at 1:1,000, toll-like receptor 2 (TLR2) at 
A

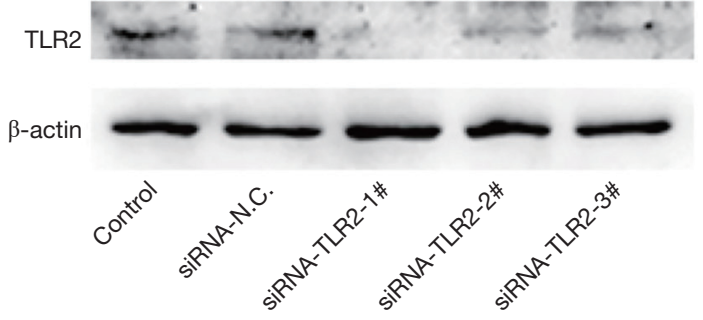

B

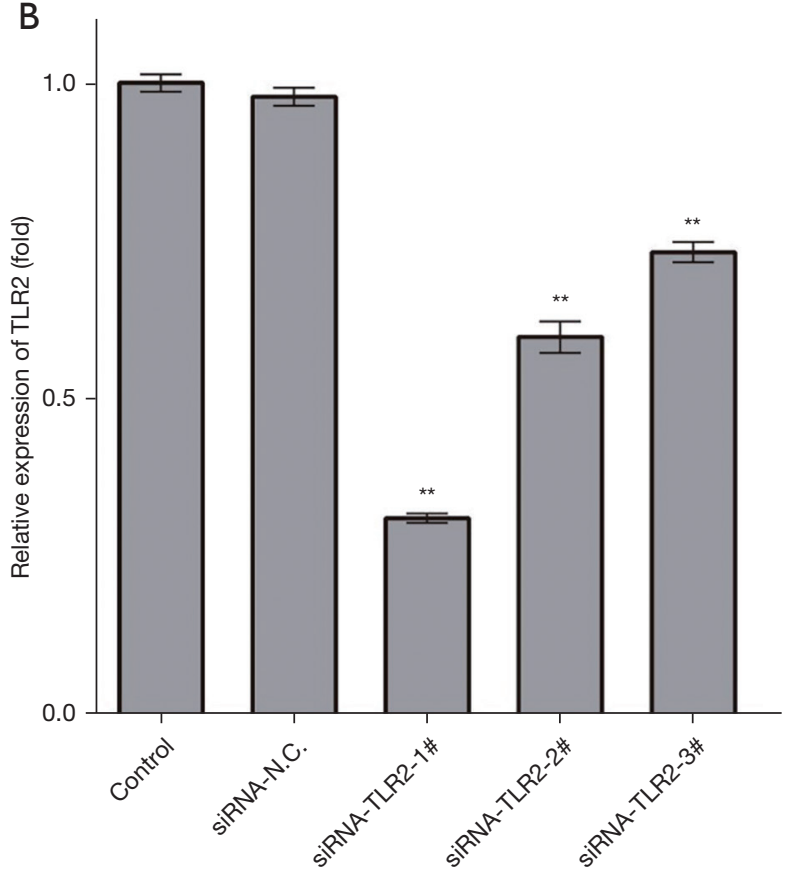

Figure 1 Specific silencing of TLR2 by siRNA in L02 cells. (A) Western blot was used to detect the effect of three TLR2-specific siRNAs on TLR2 protein expression at $24 \mathrm{~h}$ after transfection, siRNA-N.C. as a negative control for the transfection vector. (B) Histogram showing the relative TLR2 protein levels. The mean ${ }_{ \pm} \mathrm{SD}$ from triplicate independent experiments is shown. ${ }^{* *}, \mathrm{P}<0.01$ compared with siRNA-N.C. group and control group.

1:1,000, myeloid differentiation primary response gene 88 (MyD88) at 1:1,000, phospho-inhibitor of nuclear factor kappa-B kinase $\alpha(\mathrm{p}-\mathrm{IKK} \alpha)$ at $1: 1,000, \mathrm{NF}-\mathrm{\kappa B}$ at $1: 1,000$, phosphorylated-P38 (p-P38) at 1:1,000, Bcl-2 at 1:1,000, BAX at 1:1,000, and Caspase- 8 at 1:1,000), overnight at $4{ }^{\circ} \mathrm{C}$. After being washed with Tris-buffered saline ( $\mathrm{pH} 7.2)$ containing $0.05 \%$ Tween 20 (TBST), the membranes were incubated with the secondary antibody at room temperature for $1.5 \mathrm{~h}$ before being washed with TBST and incubated with the enhanced chemiluminescence reagent to detect the proteins of interest. The primary rabbit monoclonal or polyclonal antibodies for $\beta$-Actin, IRS1, PI3K, TLR2, MyD88, Bcl-2, and BAX were purchased from Proteintech (Wuhan, China), while the antibodies for AKT, p-AKT, p-IKK $\alpha$, NF- $\kappa$ B, p-P38, and Caspase- 8 were purchased from Cell Signaling Technology (Beverly, MA, USA). The levels of $\beta$-Actin were used as loading controls. The quantification of band intensity was performed by using Quantity One software (Bio-Rad, CA, USA).

\section{Statistical analysis}

GraphPad Prism 6.00 software (GraphPad Software, Inc, La Jolla, CA, USA) was used for statistical analyses. The results were expressed as the mean \pm standard deviation $(\mathrm{SD})$, and statistical significance was determined with one-way ANOVA conjunction with Bonferroni correction. A level of $\mathrm{P}<0.05$ was considered statistically significant.

\section{Results}

\section{Specific silencing of TLR2 by siRNA in L02 cells}

To inhibit TLR2, three specific target-siRNA oligonucleotides towards it were transfected in L02 cell lines, and the negative control nonsense-siRNA oligonucleotide (siRNA-N.C.) was transfected as a vectortreated control. TLR2 protein expression was analyzed by Western blot at $24 \mathrm{~h}$ after transfection (Figure $1 \mathrm{~A}$ ). Three siRNA-TLR2s protein relative expression levels were found to be significantly downregulated, compared to the untreated L02 cells and the siRNA-N.C.-treated L02 cells, respectively (Figure 1B). Further, siRNA-TLR2-1\# abolished protein expression of TLR2 more effectively than siRNA-TLR2-2\# and siRNA-TLR2-3\#, while siRNA-N. C. had no significant inhibitory effect on TLR2 protein expression. Thus, siRNA-TLR2-1\#, namely siRNA-TLR2, was used in the following experiments.

\section{TLR2 siRNA attenuated LPS-induced amplification effect on lipid accumulation in the NAFLD cell model via modulating the IRS1/PI3K/AKT signaling pathway}

Oil red $\mathrm{O}$ staining showed that lipid accumulation was significantly increased in the PA-induced NAFLD cell model, and the FFA content in the siRNA-N.C. + PA group was also significantly higher than that in the siRNA-N. 
A
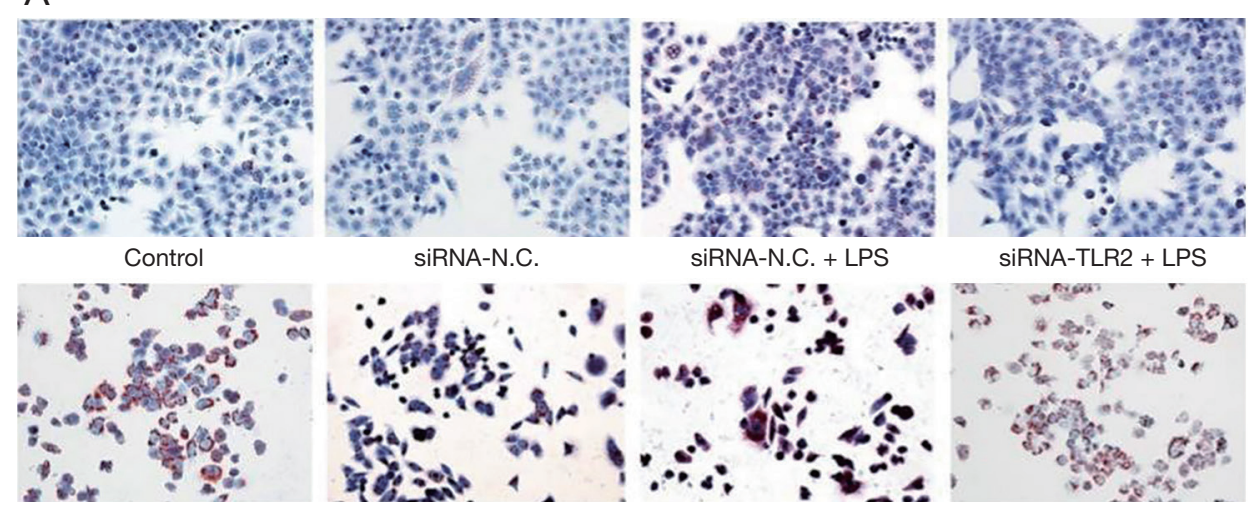

siRNA-N.C. + PA

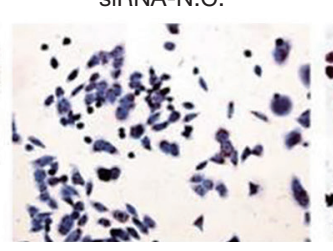

SIRNA-TLR2 + PA

C
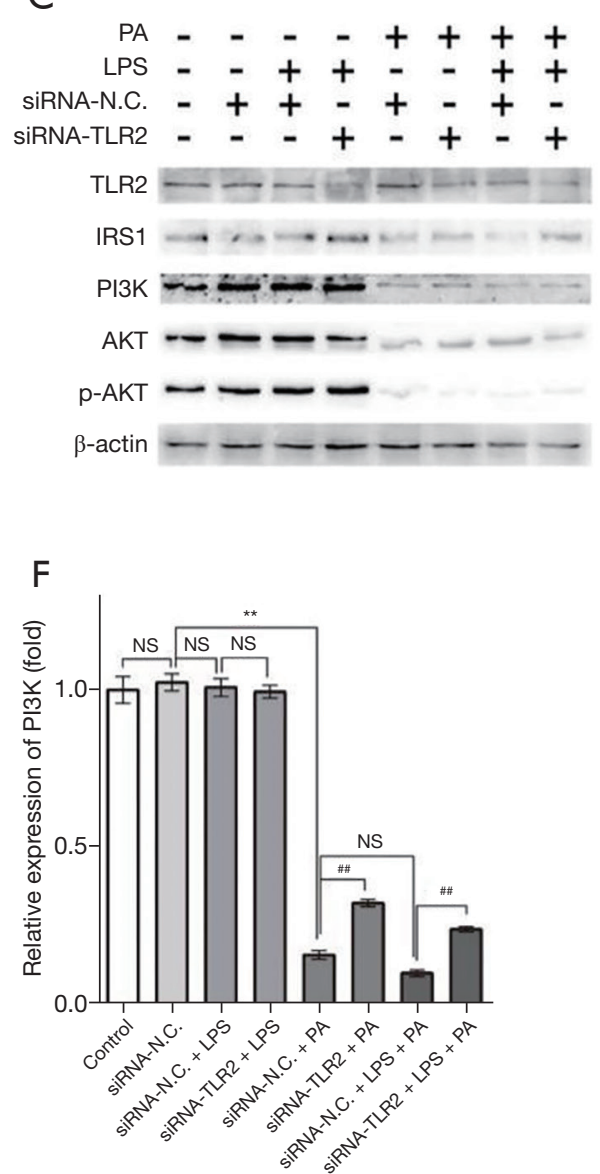
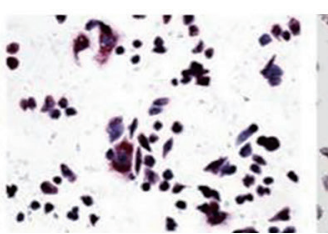

SiRNA-N.C. + LPS + PA

$\mathrm{D}$
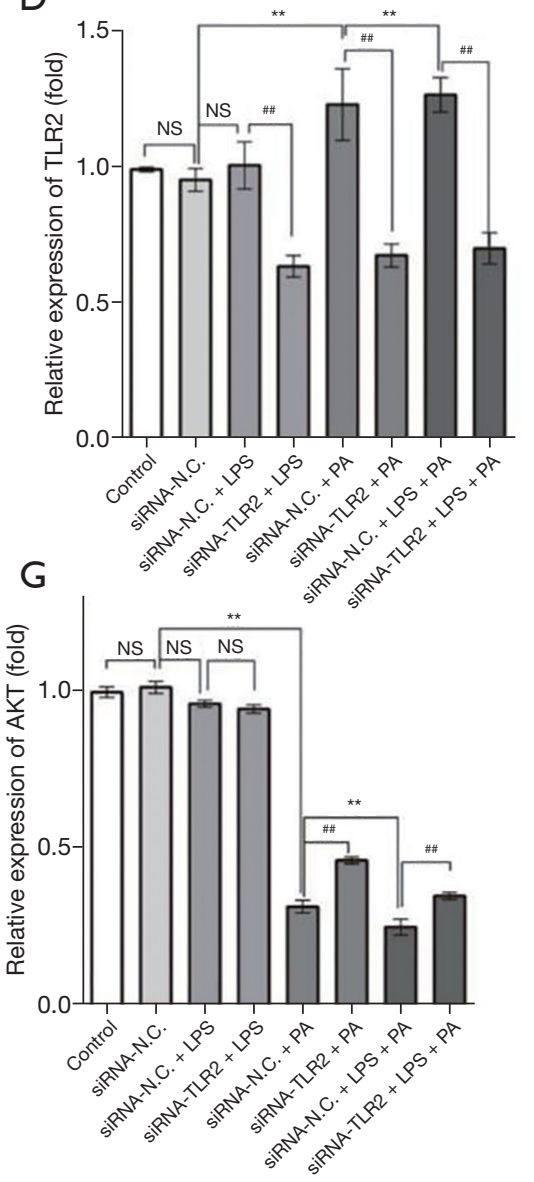

B

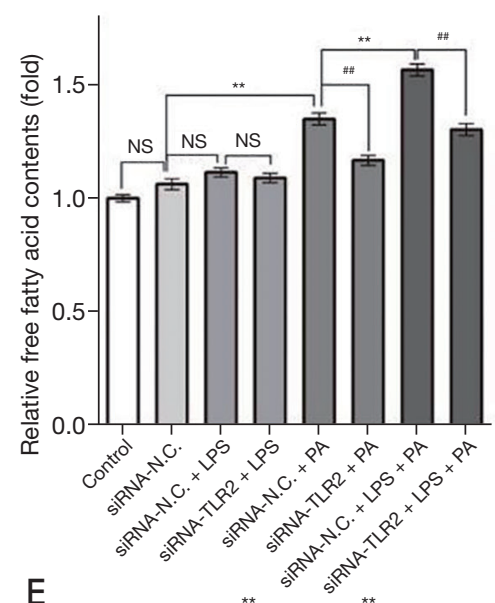

E
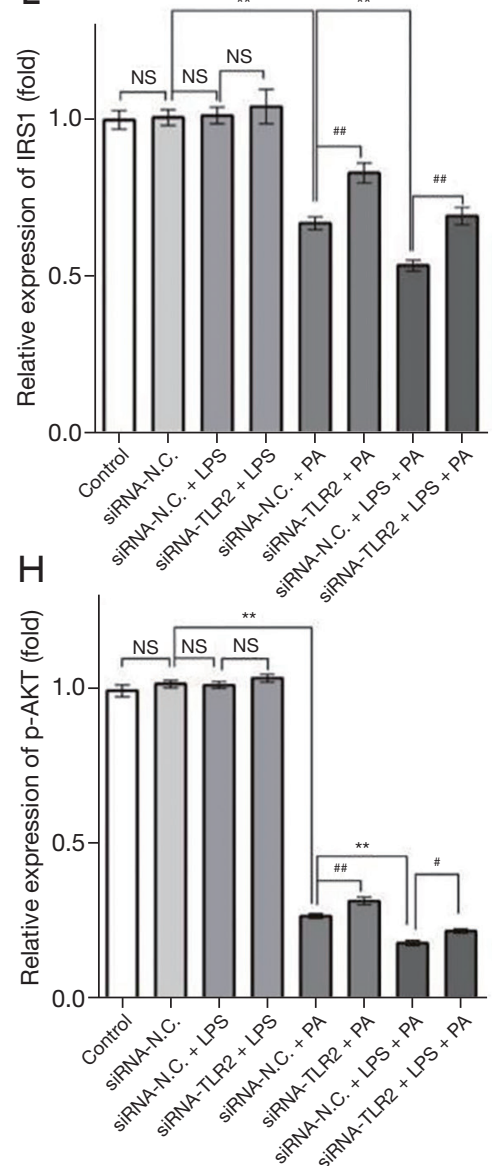

Figure 2 TLR2-siRNA attenuated LPS-induced amplification effect on lipid accumulation in the NAFLD cell model via modulating the IRS1/PI3K/AKT signaling pathway. Oil red O staining results (magnification, $\times 200$ ) (A) and the relative FFA contents (B) of L02 cells incubated with PA and/or LPS for $24 \mathrm{~h}$ after treatment with or without siRNA-N.C. or siRNA-TLR2. (C) Expression levels of TLR2, IRS1, PI3K, AKT, and p-AKT detected by Western blot. (D-H) The relative expression levels of TLR2, IRS1, PI3K, AKT, and p-AKT. The mean \pm SD from triplicate independent experiments is shown. $\mathrm{P}$ values between the groups indicated by line segments were calculated using a oneway ANOVA. **, $\mathrm{P}<0.01$;, $\mathrm{P}<0.05 ;{ }^{\text {\#\# }}, \mathrm{P}<0.01$. NS, no significance; N.C., negative control; PA, palmitic acid; LPS, lipopolysaccharide; FFA, free fatty acid. 
C. group (Figure $2 A, B)$. Moreover, FFA content in the siRNA-N.C. + LPS + PA group was markedly increased compared with the siRNA-N.C. + PA group, and the result of oil red $\mathrm{O}$ staining was in line with this result, suggesting LPS could enhance lipid accumulation in the NAFLD cell model. Further, lipid accumulation and FFA content were attenuated largely after siRNA-TLR2 treatment (siRNATLR2 + PA group vs. siRNA-N.C. + PA group; siRNATLR2 + LPS + PA group vs. siRNA-N.C. + LPS + PA group), suggesting TLR2 inhibition had a protective effect against PA-induced and LPS-amplified lipid accumulation. Under the effect of LPS alone, FFA content showed a slight increase, and there was no statistical significance (siRNAN.C. + LPS group vs. siRNA-N.C. group), and when TLR2 was inhibited, LPS had no significant effect on FFA content (siRNA-TLR2 + LPS group vs. siRNA-N.C. + LPS group).

To study the possible molecular mechanisms of the effect of TLR2 and LPS on lipid accumulation in the NAFLD cell model, we detected the expression of proteins in the IRS1/PI3K/AKT signaling pathway, which was associated with lipid accumulation (Figure $2 \mathrm{C}-2 \mathrm{H}$ ). In the PA-induced NAFLD cell model, the expression of IRS1, PI3K, AKT, and p-AKT decreased significantly, whereas the level of TLR2 increased remarkably (siRNA-N.C. + PA group $v$. siRNA-N.C. group). In addition, LPS enlarged the trend of decreased IRS1, PI3K, AKT, and p-AKT, and increased TLR2 (siRNA-N.C. + LPS + PA group vs. siRNA-N.C. + $\mathrm{PA}$ group). These results implied that lipid accumulation may be related to inhibition of the IRS1/PI3K/AKT signaling pathway in the PA-induced NAFLD cell model, and LPS aggravates lipid accumulation with its further inhibition. At the same time, TLR2 increased with relevant levels in these processes, indicating it may play a key role in PA-induced and LPS-amplified lipid accumulation. Furthermore, TLR2 inhibition partially recovered the levels of IRS1, PI3K, AKT, and p-AKT in the NAFLD cell model and LPS-treated NAFLD cell model (siRNA-TLR2 + PA group vs. siRNA-N.C. + PA group; siRNA-TLR2 + LPS + PA group vs. siRNA-N.C. + LPS + PA group), and LPS had no significant effect on the IRS1/PI3K/AKT signaling pathway when TLR2 expressed normally or lowly (siRNA-N.C. + LPS group vs. siRNA-N.C. group; siRNATLR2 + LPS group vs. siRNA-N.C. + LPS group). Based on these results, we believe TLR2 siRNA attenuated PAinduced and LPS-amplified lipid accumulation via activating the IRS1/PI3K/AKT signaling pathway.

\section{TLR2 siRNA alleviated LPS-induced amplification effect on inflammatory response in the NAFLD cell model through targeting the TLR2/MyD88/IKKa/NF- $B$ signaling patbway}

Secreted pro-inflammatory cytokines are the response to lipotoxicity in the NAFLD model. In this work, we detected the levels of IL- 6 and TNF- $\alpha$ by ELISA (Figure $3 A, 3 B$ ), and found PA and LPS significantly promoted IL-6 secretion levels compared with control, respectively (siRNA-N.C. + PA group vs. siRNA-N.C. group; siRNA-N.C. + LPS group $v s$. siRNA-N.C. group), and o-treatment with LPS and PA had an additive effect (siRNA-N.C. + LPS + PA group $v s$. siRNA-N.C. group). The results indicated LPS aggravated the levels of IL-6 in PA-induced the NAFLD cell model. Furthermore, we found that the inhibition of TLR2 could effectively reduce the secreted IL-6 in the NAFLD cell model (siRNA-TLR2 + PA group vs. siRNA-N.C. + PA group; siRNA-TLR2 + LPS + PA group vs. siRNA-N.C. + LPS + PA group), but not in L02 cells treated with LPS only (siRNA-TLR2 + LPS group vs. siRNA-N.C. + LPS group), indicating TLR2 inhibition alleviates PA-induced rather than LPS-induced inflammation in L02 cells. For TNF- $\alpha$, a similar trend to IL- 6 was observed in groups, but there was no significant change.

To clarify the underlying mechanisms by which TLR2 siRNA treatment exerted its anti-inflammatory effect in the PA-induced NAFLD cell model, MyD88, p-IKK $\alpha$, and $\mathrm{NF}-\kappa \mathrm{B}$ were examined by Western blot. As shown in Figure 3C-3G, PA significantly up-regulated the expression of MyD88, p-IKK $\alpha$, and NF- $\kappa B$ in the siRNA-N.C. + PA group compared with siRNA-N.C. group. When co-treated with LPS and PA, the expression of MyD88, p-IKK $\alpha$, and $\mathrm{NF}-\kappa \mathrm{B}$ were further increased (siRNA-N.C. + LPS + PA group $v s$. siRNA-N.C. group), and while knockdown of TLR2 showed the opposite results in the NAFLD cell model (siRNA-TLR2 + PA group vs. siRNA-N.C. + PA group; siRNA-TLR2 + LPS + PA group vs. siRNA-N.C. + LPS + PA group), no significant changes were observed in L02 cells treated with LPS only (siRNA-TLR2 + LPS group vs. siRNA-N.C. + LPS group). We further quantified the p-P38 in groups, which was up-regulated under oxidative stress caused by inflammation, lipotoxicity, or other stress, and found PA could induce remarkably elevated p-P38 expression, and co-treatment with LPS and PA facilitated this. After siRNA-TLR2 treatment, p-P38 expression was partially down-regulated when cells were stimulated with 
A

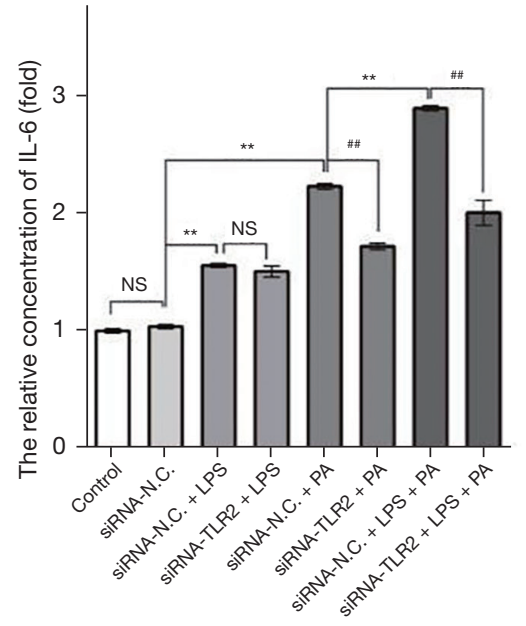

B

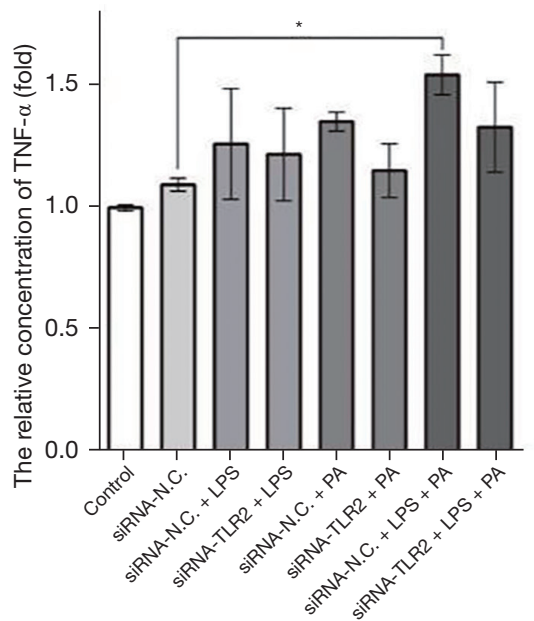

C

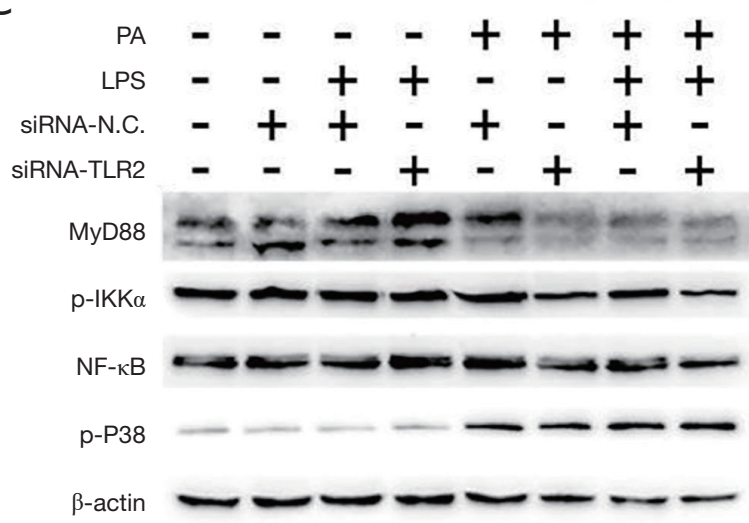

$\mathrm{E}$

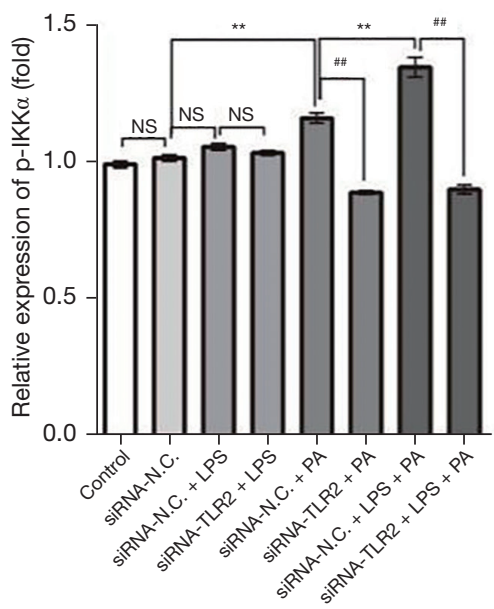

$\mathrm{F}$

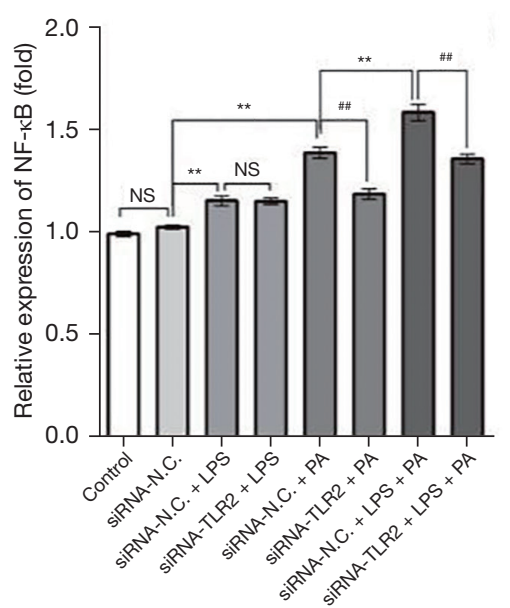

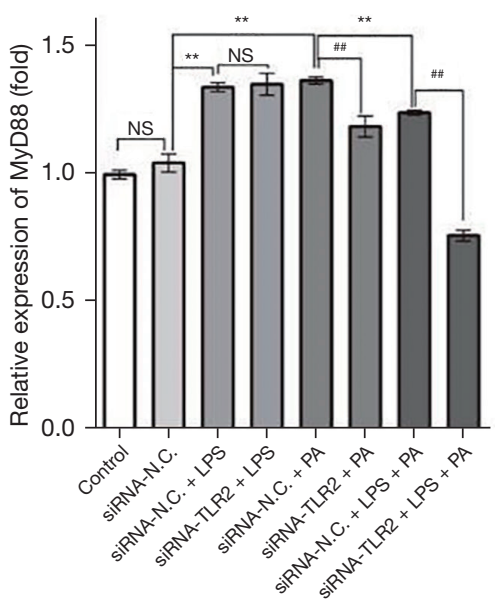

G

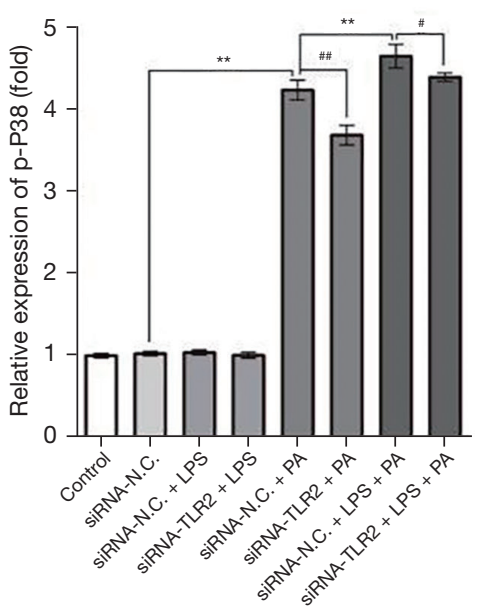

Figure 3 TLR2-siRNA alleviated LPS-induced amplification effect on inflammatory response in the NAFLD cell model through targeting the TLR2/MyD88/IKKa/NF-KB signaling pathway. Release levels of IL-6 (A) and TNF- $\alpha$ (B) of L02 cells incubated with PA and/ or LPS for $24 \mathrm{~h}$ after treatment with or without siRNA-N.C. or siRNA-TLR2. (C) Expression levels of MyD88, p-IKK $\alpha$, NF- $\kappa B$, and p-P38 detected by Western blot. (D-G) Relative expression levels of MyD88, p-IKKa, NF- $\kappa B$, and p-P38. The mean \pm SD from triplicate independent experiments is shown. $\mathrm{P}$ values between the groups indicated by line segments were calculated using a one-way ANOVA. *, $\mathrm{P}<0.05 ;{ }^{* *}, \mathrm{P}<0.01 ;{ }^{\#}, \mathrm{P}<0.05 ;$;, $\mathrm{P}<0.01$. NS, no significance; N.C., negative control; PA, palmitic acid; LPS, lipopolysaccharide. 
PA only or co-treatment with PA and LPS, but not LPS alone. These results indicate LPS aggravates PA-induced inflammation in L02 cells, and TLR2 siRNA alleviates PAinduced rather than LPS-induced inflammation through targeting the TLR2/MyD88/IKK $\alpha / \mathrm{NF}-\kappa \mathrm{B}$ signaling pathway.

\section{TLR2 siRNA ameliorated LPS-induced amplification effect on cell apoptosis in the NAFLD cell model by regulating the mitochondrion-dependent apoptotic signaling patbway}

To investigate the effect of LPS on cell apoptosis in the PAinduced NAFLD cell model, Hoechst 33342 staining and Caspase-3 activity assays were performed (Figure $4 A, 4 B$ ). The results showed that compared with the siRNA-N.C. group, Caspase-3 activity in the siRNA-N.C. + PA group was significantly increased but not in the siRNA-N.C. + LPS group, as demonstrated by the increased nuclear condensation ratio. Furthermore, Caspase- 3 activity and the ratio of nuclear condensation in the siRNA-N.C. + LPS + PA group were higher than those in the siRNA-N.C. + PA group, suggesting LPS amplificated cell apoptosis in the NAFLD cell model. However, Caspase-3 activity and the ratio of nuclear condensation were ameliorated distinctly by TLR2 knockdown when cells were stimulated with PA only or with PA and LPS co-treatment (siRNA-TLR2 + PA group vs. siRNA-N.C. + PA group, siRNA-TLR2 + LPS + PA group $v s$. siRNA-N.C. + LPS + PA group), but not with LPS alone (siRNA-TLR2 + LPS group vs. siRNA-N.C. + LPS group), suggesting TLR2 silencing may prevent the LPS-induced amplification effect on cell apoptosis in the NAFLD cell model.

To further determine the role of the mitochondriondependent apoptotic signaling pathway on LPS-induced cell apoptosis in the NAFLD cell model, we explored the expression pattern of Bcl-2, BAX, and Caspase- 8 in the LPS-exposed NAFLD cell model (Figure $4 C-4 F$ ). In the PA-induced NAFLD cell model, BAX and cleaved Caspase- 8 increased by 1.59 -fold and 1.41-fold, respectively, but had no effect on Bcl-2 expression (siRNA-N.C. + PA group $v s$. siRNA-N.C. group). However, when the NAFLD cell model was exposed to LPS, the up-regulation of BAX and cleaved Caspase- 8 robustly increased compared with the NAFLD model cells, while the expression of Bcl2 decreased sharply (siRNA-N.C. + LPS + PA group vs. siRNA-N.C. + PA group). These results suggest the mitochondrion-dependent apoptotic signaling pathway is implicated in the LPS-mediated amplification effect on PA- induced cell apoptosis in L02 cells. Furthermore, TLR2 siRNA treatment partially diminished the PA-induced and LPS-amplified increased expression of BAX and cleaved Caspase-8, as well as PA-induced and LPS-suppressed decreased expression of Bcl-2 in the NAFLD cell model (siRNA-TLR2 + PA group vs. siRNA-N.C. + PA group, siRNA-TLR2 + LPS + PA group vs. siRNA-N.C. + LPS + PA group), but not in L02 cells treated with LPS only (siRNA-TLR2 + LPS group $v s$. siRNA-N.C. + LPS group). Taken together, the results suggest LPS had no significant effect on the mitochondrion-dependent apoptotic signaling pathway when TLR2 expressed normally or lowly, but TLR2 siRNA ameliorated PA-induced and LPS-amplified cell apoptosis in the NAFLD cell model by regulating the mitochondrion-dependent apoptotic signaling pathway.

\section{Discussion}

In the present study, a PA-induced NAFLD cell model was used to explore the role of LPS and TLR2 in the development of NAFLD. Data showed that LPS aggravated lipid accumulation and lipotoxicity through inhibiting the IRS1/PI3K/AKT signaling pathway, activating the TLR2/ MyD88/IKK $\alpha / \mathrm{NF}-\mathrm{kB}$ signaling pathway, and activating the mitochondrion-dependent apoptotic signaling pathway in PA-treated L02 cells.

In the "Two-Hit Hypothesis" of the pathogenesis of NAFLD, IR is described as the "first hit", which contributes to lipid accumulation $(2,14,15)$. Previous studies suggest the IRS1/PI3K/AKT signaling pathway is associated with the pathogenesis of NAFLD. Zhang et al. found IRS1, $\mathrm{PI} 3 \mathrm{~K}$, and $\mathrm{p}-\mathrm{AKT}$ were down-regulated in the NAFLD rat model (14), while Xiao et al. found PI3K and AKT activation could protect the liver from NASH-induced injury (16). In the present study, a significant increase in lipid accumulation and FFA content was accompanied by the decreased expression of IRS1, PI3K, AKT, and $\mathrm{p}$-AKT in the PA-induced NAFLD cell model, whereas the protein expression of TLR2 was remarkably increased. LPS enhanced lipid accumulation in the NAFLD cell model, with an enlarged trend of decreased IRS1, PI3K, AKT, $\mathrm{p}-\mathrm{AKT}$, and increased TLR2. Hence, we assume that the lipid accumulation induced by PA may be related to IR, and LPS may aggravate IR-related lipid accumulation.

It has been reported that TLR2 and PA cooperatively activate the inflammasome involved in the progression of NASH (3), and here, we also evaluated the levels of proinflammatory cytokines and the TLR2/MyD88/IKK $\alpha / \mathrm{NF}$ - 


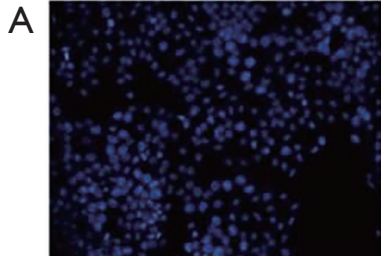

Control

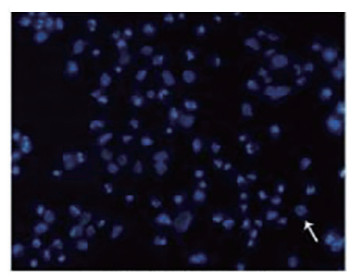

siRNA-N.C. + PA

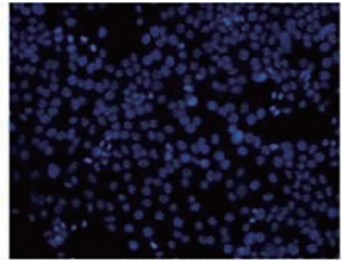

SiRNA-N.C.

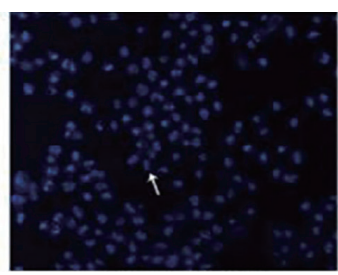

SiRNA-TLR2 + PA

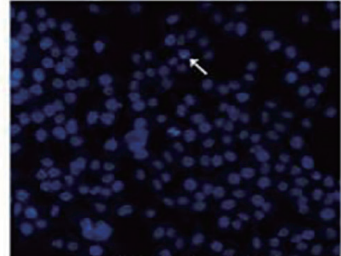

SiRNA-N.C. + LPS

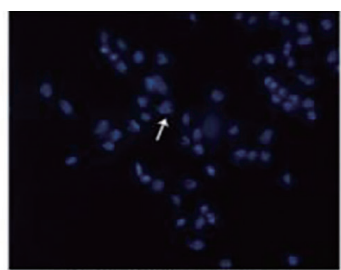

siRNA-N.C. + LPS + PA

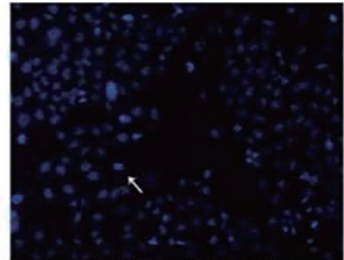

SiRNA-TLR2 + LPS

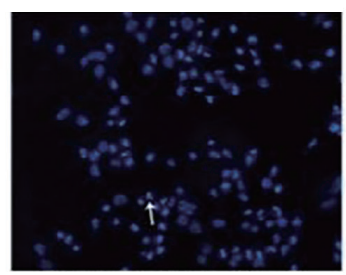

SiRNA-TLR2 + LPS + PA
B

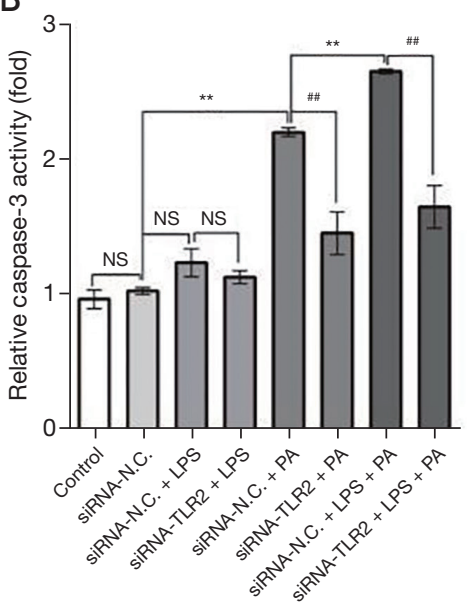

D

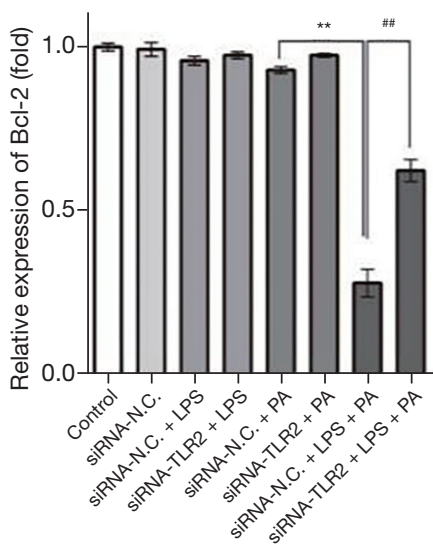

C

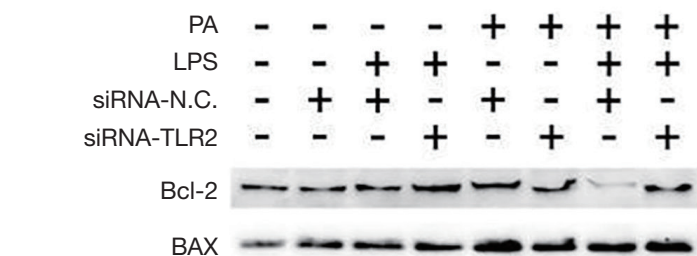

Cleaved caspase-8 $\quad-\ldots$

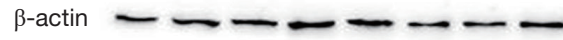

$\mathrm{E}$

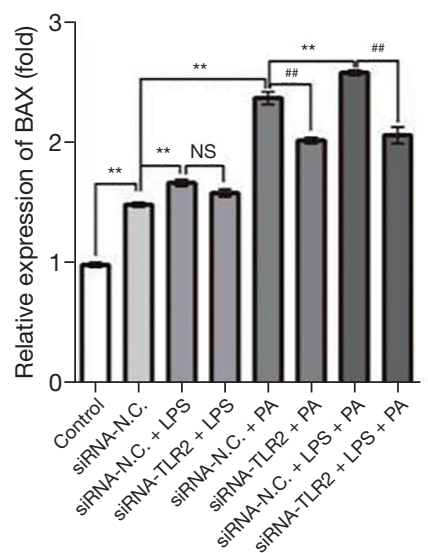

$\mathrm{F}$

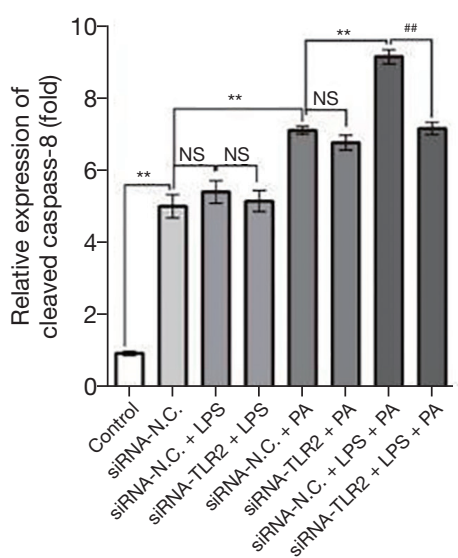

Figure 4 TLR2-siRNA ameliorated LPS-induced amplification effect on cell apoptosis in the NAFLD cell model by regulating the mitochondrion-dependent apoptotic signaling pathway. Hoechst 33342 staining results (magnification, $\times 200$ ) (A) (white arrow stands for apoptotic bodies) and Caspase-3 activity (B) of L02 cells incubated with PA and/or LPS for $24 \mathrm{~h}$ after treatment with or without siRNA-N. C. or siRNA-TLR2. (C) Expression levels of Bcl-2, BAX, and cleaved Caspase-8 detected by Western blot. (D-F) Relative expression levels of Bcl-2, BAX, and cleaved Caspase- 8 . The mean \pm SD from triplicate independent experiments is shown. P-values between the groups indicated by line segments were calculated using a one-way ANOVA. **, $\mathrm{P}<0.01$; ${ }^{\# \#}, \mathrm{P}<0.01$. NS, no significance; N.C., negative control; PA, palmitic acid; LPS, lipopolysaccharide. 
$\kappa \mathrm{B}$ signaling pathway. The results showed that $\mathrm{PA}$ and/or LPS increased IL6 and TNF- $\alpha$, especially IL6, and with TLR2, MyD88, p-IKKa, and NF-кB up-regulation, TLR2 was not significantly up-regulated in cells treated with LPS alone. However, the amplification effect of LPS on PAinduced inflammation was still observed. In accordance with our results, increased levels of pro-inflammatory cytokines promoted liver injury in NAFLD mice injected with LPS (17). LPS was also reported to induce high levels of FFA and lipid accumulation $(13,18)$, whereas saturated fatty acids could up-regulate inflammasome and induce hepatocyte sensitivity to LPS in NASH mice (19), and circulating endotoxin levels were also higher in NAFLD patients (20). The "vicious cycle" relationship between LPS and lipotoxicity may represent the mechanism by which gut microbiota affects the progression of NAFLD through the gut-liver axis (5). Gut microbiome dysbiosis affects inflammation levels, and NF- $\mathrm{KB}$ activation seems to promote hepatic gluconeogenesis, which also links liver inflammation and IR $(5,21)$. The "vicious cycle" includes inflammation, lipotoxicity, and IR, and can result in elevated oxidative stress $(2,6)$. The levels of p-P38 also reflected that LPS aggravated PA-induced oxidative stress in this study. The results suggest that blocking the "vicious cycle" may provide new insights into prevention and treatment strategies for NAFLD.

Hepatocyte lipoapoptosis is characteristic of NAFLD, and can be caused by endoplasmic reticulum stress, oxidative stress, and mitochondrial dysfunction, involving the Jun $\mathrm{N}$-terminal kinase signaling pathway and mitochondrial apoptotic pathway $(2,22)$. In the present study, an increased nuclear condensation ratio was accompanied by increased caspase- 3 activity, BAX expression, and cleaved caspase- 8 expression, but had no effect on Bcl-2 expression in the PAinduced NAFLD cell model. LPS-induced an amplification effect on the increased nuclear coagulation rate, caspase- 3 activity, BAX expression, and cleaved caspase- 8 expression, while the expression of Bcl-2 decreased sharply. Thus, the mitochondrion-dependent apoptotic signaling pathway is implicated in the amplification effect caused by LPS on PAinduced cell apoptosis.

The effects of siRNA-mediated TLR2 silencing on these signaling pathways were further studied. We found that TLR2 siRNA could alleviate the lipid accumulation and lipotoxicity induced by PA with or without LPS, and alleviate the pathway of inhibition or activation, but had no significant effect on L02 cells treated with LPS only. In our previous study, TLR4 was involved in the amplification of LPS in PA-treated L02 cells (13), and in line with our results, serum TLR4 levels but not TLR2 were elevated in NASH patients (23). However, it has been reported that TLR2 could synergize with PA to activate inflammasome involved in the progression of NASH (3). Although TLR2 is the target receptor of Gram-positive bacteria, some studies suggest it could sense the stimulation of LPS $(10,12)$. While Furusho et al. believed that LPS from Porphyromonas gingivalis up-regulated TLR2 to promote NASH progression (12). This may be due to the unusual nature of this bacteria, as it synthesizes complex lipids other than LPS, which may contribute to TLR2 activation (24). LPS from Escherichia coli was used in our study, because it is the most abundant species in patients with NAFLDrelated advanced fibrosis (25). Considering the complexity of gut microbiome, the specific role of TLR2 in the gut microbiome-associated NAFLD is still worth studying.

In conclusion, this study revealed that lipid accumulation and lipotoxicity could be aggravated by LPS in a PA-induced NAFLD cell model, and the molecular mechanism involves the IRS1/PI3K/AKT signaling pathway, TLR2/MyD88/ IKK $\alpha / N F-\kappa B$ signaling pathway, and mitochondriondependent apoptotic signaling pathway. During this process, TLR2 can be activated by PA, but not by LPS. These data provide a perspective for understanding the mechanism of gut microbiome promoting the development of NAFLD.

\section{Acknowledgments}

Funding: This study was supported by the National Natural Science Foundation of China (grant No. 81360141), the Postdoctoral Science Foundation of China (grant no. 22019M653474), the National Natural Science Foundation (81800528), Natural Science Foundation of Gansu Province (20JR5RA364), Key Research and Development Project of Gansu Province (20YF2FA011), and the Health Industry Research Project in Gansu Province (GSWSKY2018-24), Natural Science Foundation of Gansu Province (20JR0RA683), Intra-hospital Fund of the First Hospital of Lanzhou University (LDYYYN2018-41).

\section{Footnote}

Reporting Checklist: The authors have completed the MDAR reporting checklist. Available at https://dx.doi. org/10.21037/atm-21-4012

Data Sharing Statement: Available at https://dx.doi. 
org/10.21037/atm-21-4012

Conflicts of Interest: All authors have completed the ICMJE uniform disclosure form (available at https://dx.doi. org/10.21037/atm-21-4012). The authors have no conflicts of interest to declare.

Etbical Statement: The authors are accountable for all aspects of the work in ensuring that questions related to the accuracy or integrity of any part of the work are appropriately investigated and resolved.

Open Access Statement: This is an Open Access article distributed in accordance with the Creative Commons Attribution-NonCommercial-NoDerivs 4.0 International License (CC BY-NC-ND 4.0), which permits the noncommercial replication and distribution of the article with the strict proviso that no changes or edits are made and the original work is properly cited (including links to both the formal publication through the relevant DOI and the license). See: https://creativecommons.org/licenses/by-nc-nd/4.0/.

\section{References}

1. Wong VW, Chitturi S, Wong GL, et al. Pathogenesis and novel treatment options for non-alcoholic steatohepatitis. Lancet Gastroenterol Hepatol 2016;1:56-67.

2. Engin A. Non-Alcoholic Fatty Liver Disease. Adv Exp Med Biol 2017;960:443-67.

3. Yoneda M, Ogawa Y, Imajo K, et al. Nonalcoholic fatty liver disease with advanced fibrosis as a multi-systemic disease: proceed with caution. Hepatobiliary Surg Nutr 2019;8:170-2.

4. Marra F, Svegliati-Baroni G. Lipotoxicity and the gut-liver axis in NASH pathogenesis. J. Hepatol. 2018;68:280-95.

5. Ma J, Zhou Q, Li H. Gut Microbiota and Nonalcoholic Fatty Liver Disease: Insights on Mechanisms and Therapy. Nutrients 2017;9:1124.

6. Zhang X, Ji X, Wang Q, et al. New insight into interorgan crosstalk contributing to the pathogenesis of nonalcoholic fatty liver disease (NAFLD). Protein Cell 2018;9:164-77.

7. Acharya C, Bajaj JS. Altered Microbiome in Patients With Cirrhosis and Complications. Clin Gastroenterol Hepatol 2019;17:307-21.

8. Moreno-Gonzalez M, Beraza N. The Role of the Microbiome in Liver Cancer. Cancers (Basel) 2021;13:2330.
9. Lang S, Schnabl B. Microbiota and Fatty Liver Diseasethe Known, the Unknown, and the Future. Cell Host Microbe 2020;28:233-44.

10. Paolella G, Mandato C, Pierri L, et al. Gut-liver axis and probiotics: their role in non-alcoholic fatty liver disease. World J Gastroenterol 2014;20:15518-31.

11. Szabo G, Velayudham A, Romics L Jr, et al. Modulation of non-alcoholic steatohepatitis by pattern recognition receptors in mice: the role of toll-like receptors 2 and 4. Alcohol Clin Exp Res 2005;29:140S-5S.

12. Furusho H, Miyauchi M, Hyogo H, et al. Dental infection of Porphyromonas gingivalis exacerbates high fat diet-induced steatohepatitis in mice. J. Gastroenterol 2013;48:1259-70.

13. Li Y, Wang C, Lu J, et al. PPAR $\delta$ inhibition protects against palmitic acid-LPS induced lipidosis and injury in cultured hepatocyte L02 cell. Int J Med Sci 2019;16:1593-603.

14. Zhang Y, Hai J, Cao M, et al. Silibinin ameliorates steatosis and insulin resistance during non-alcoholic fatty liver disease development partly through targeting IRS-1/PI3K/ Akt pathway. Int Immunopharmacol 2013;17:714-20.

15. Gillessen A, Schmidt HH. Silymarin as Supportive Treatment in Liver Diseases: A Narrative Review. Adv Ther 2020;37:1279-301.

16. Xiao J, Liong EC, Ching YP, et al. Lycium barbarum polysaccharides protect rat liver from non-alcoholic steatohepatitis-induced injury. Nutr. Diabetes 2013;3:e81.

17. Perea L, Coll M, Sanjurjo L, et al. Pentraxin-3 modulates lipopolysaccharide-induced inflammatory response and attenuates liver injury. Hepatology 2017;66:953-68.

18. Hernández EÁ, Kahl S, Seelig A, et al. Acute dietary fat intake initiates alterations in energy metabolism and insulin resistance. J Clin Invest 2017;127:695-708.

19. Csak T, Ganz M, Pespisa J, et al. Fatty acid and endotoxin activate inflammasomes in mouse hepatocytes that release danger signals to stimulate immune cells. Hepatology 2011;54:133-44.

20. Mohammad S, Thiemermann C. Role of Metabolic Endotoxemia in Systemic Inflammation and Potential Interventions. Front.Immunol 2021;11:594150.

21. Okin D, Medzhitov R. The Effect of Sustained Inflammation on Hepatic Mevalonate Pathway Results in Hyperglycemia. Cell 2016;165:343-56.

22. Leamy AK, Egnatchik RA, Young JD. Molecular mechanisms and the role of saturated fatty acids in the progression of non-alcoholic fatty liver disease. Prog. Lipid Res 2013;52:165-74. 
23. Cengiz M, Ozenirler S, Elbeg S. Role of serum toll-like receptors 2 and 4 in non-alcoholic steatohepatitis and liver fibrosis. J.Gastroenterol.Hepatol 2015;30:1190-6.

24. Jain S, Darveau RP. Contribution of Porphyromonas gingivalis lipopolysaccharide to periodontitis. Periodontol
2000 2010;54:53-70.

25. Loomba R, Seguritan V, Li W, et al. Gut MicrobiomeBased Metagenomic Signature for Non-invasive Detection of Advanced Fibrosis in Human Nonalcoholic Fatty Liver Disease. Cell Metab 2017;25:1054-1062.e5.

Cite this article as: Zhang $\mathrm{L}, \mathrm{Xie} \mathrm{Z}$, $\mathrm{Yu} \mathrm{H}, \mathrm{Du} \mathrm{H}$, Wang $\mathrm{X}$, Cai J, Qiu Y, Chen R, Jiang X, Liu Z, Li Y, Chen T. TLR2 inhibition ameliorates the amplification effect of LPS on lipid accumulation and lipotoxicity in hepatic cells. Ann Transl Med 2021;9(18):1429. doi: 10.21037/atm-21-4012 